\title{
TRANSPLANTATION
}

\section{Tofacitinib safe and effective in renal transplant recipients}

The oral Janus kinase inhibitor, tofacitinib, is safe and effective in low-to-moderate risk de novo renal transplant recipients when compared with ciclosporin shows a new phase $2 \mathrm{~b}$ study published in the American Journal of Transplantation.

"Rejection rates were low with better preservation of renal function, so these results are potentially an important advance for calcineurin-inhibitor-free regimens", says researcher Flavio Vincenti.

Although calcineurin inhibition remains the cornerstone of immunosuppressive regimens after transplantation, alternative drugs with fewer adverse effects are being investigated. Tofacitinib suppresses intracellular signal transduction of multiple cytokines and has been shown in a pilot study to prevent acute rejection.

In their multicenter trial, Vincenti et al. randomly assigned 331 patients to a more intensive or less intensive regimen of tofacitinib or to ciclosporin. All patients received basiliximab induction, mycophenolic acid and corticosteroids. The primary end points were the
4 Both tofacitinib regimens were noninferior compared with ciclosporin for the primary efficacy end point... 77

incidence of biopsy-proven acute rejection at month 6 with an increase in serum creatinine levels and measured glomerular filtration rate (GFR) at month 12 .

Both tofacitinib regimens were noninferior compared with ciclosporin for the primary efficacy end point of 6-month incidence of biopsy-proven acute rejection. Higher estimated GFR values were observed as early as 1 month post-transplantation in patients receiving tofacitinib than in those receiving ciclosporin, however, and renal function remained significantly higher at month 12 in the tofacitinib groups. Fewer patients developed chronic allograft nephropathy (CAN) and fewer patients showed an increase in interstitial fibrosis and/ or tubular atrophy in the tofacitinib groups. No difference in patient survival was observed between any of the groups. However, serious infections and hematological abnormalities, including anemia, neutropenia and post-transplant lymphoproliferative disorder, occurred more frequently in the tofacitinib groups than in the ciclosporin group, and optimal use of tofacitinib in transplantation may require therapeutic drug monitoring. "Adjustments to the tofacitinib regimens used in this study may permit the benefits of improved GFR and less CAN without over-immunosuppression", write the authors. "We hope that we can now move forward with a phase 3 trial as we need calcineurin-free regimens to improve long-term outcomes", concludes Vincenti.

Helene Myrvang

Original article Vincenti, F. et al. Randomized phase $2 \mathrm{~b}$ trial of tofacitinib (CP-690,550) in de novo kidney transplant patients: efficacy, renal function and safety at 1 year. Am. J. Transplant. doi:10.1111/j.16006143.2012.04127.x 